\title{
Auswirkungen der Wirtschaftskrise in Deutschland und den Niederlanden aus Sicht der Beschäftigten
}

\author{
Reinhard Bispinck, Heiner Dribbusch, Fikret Öz, \\ Kea Tijdens, Maarten van Klaveren
}

Obwohl die Krise in den Niederlanden vergleichsweise mehr Betriebe erfasst hat als in Deutschland, stellen Beschäftigte dort seltener Personalmaßnahmen sowie krisenbedingte Einkommensverluste fest. Dies ist ein Ergebnis eines Zwei-Ländervergleichs zu den Auswirkungen der jüngsten Wirtschaftskrise, der - anders als in den meisten vorliegenden Studien - die Einschätzung der Beschäftigten selbst zum Ausgangspunkt der Analyse macht. Grundlage ist ein Datensatz, der auf einer in den Niederlanden wie in Deutschland parallel durchgeführten Online-Erhebung basiert. Sie wurde im Rahmen des internationalen WageIndicator-Projekts durchgeführt.

\section{Wirtschaftskrise - einige vergleichende Kennziffern}

Deutschland und die Niederlande haben die jüngste Wirtschaftskrise unterschiedlich erfahren und verarbeitet. Die nachstehenden Kennziffern (Abbildung 1) verdeutlichen, dass der Kriseneinbruch gemessen an der Entwicklung des realen Bruttoinlandsprodukts in Deutschland deutlich kräftiger war als in den Niederlanden. Die Entwicklung am Arbeitsmarkt verlief jedoch nicht parallel: In Deutschland war die Arbeitslosenquote während des Jahres 2008 noch leicht rückläufig, stieg dann (nur) in den ersten sieben Monaten des Jahres 2009 an, um anschließend unter das Krisenausgangsniveau zurückzugehen. In den Niederlanden stieg die Arbeitslosenquote dagegen während des gesamten Krisenverlaufs kontinuierlich an, liegt aber im Niveau immer noch unter der deutschen Quote. Diese unterschiedliche Arbeitsmarktentwicklung spiegelt sich auch in der Entwicklung der Arbeitsproduktivität wieder, die in Deutschland noch deutlich unter dem Niveau zu Krisenbeginn liegt. Demzufolge hat die Entwicklung der Lohnstückkosten in den Niederlanden einen aus Sicht der Unternehmen günstigeren Verlauf genommen. Wie sich diese Entwicklung aus Sicht der Beschäftigten vollzogen hat, soll im Folgenden auf Basis einer über zehn Monate hinweg in beiden Ländern durchgeführten OnlineErhebung untersucht werden.

\section{2 \\ Anlage und Durchführung der Erhebung}

Mittlerweile gibt es eine ganze Reihe von Untersuchungen zur Wirtschafts- und Finanzkrise in verschiedenen Ländern. Die meisten dieser Studien haben die MakroEbene im Blick, selten untersucht ist hingegen, wie sich die Krise auf die Beschäftigten ausgewirkt hat. Der folgende Artikel widmet sich dieser Forschungslücke. Er untersucht in einem Zweiländervergleich Reichweite und Auswirkungen der Krise, wie sie sich in der Wahrnehmung von Beschäftigten in beiden Ländern widerspiegeln. Ausgangspunkt sind dabei persönliche Einschätzungen zur wirtschaftlichen Entwicklung und Beschäftigungssituation. Das besondere Augenmerk der Studie liegt darauf, herauszuarbeiten, welche Krisen-Auswirkungen die Beschäftigten in Bezug auf Personalabbau, Arbeitsbedingungen und Einkommen beobachteten.

Die Datenbasis stammt aus zwei vergleichbaren, kontinuierlichen Online-Befragungen, der Lohnspiegel-Umfrage in Deutschland und dem Loonwijzer-Sur- vey in den Niederlanden. Beide Umfragen zielen auf die Erhebung von Daten zur tatsächlichen Entlohnung von Beschäftigten. Dies ist verbunden mit Fragen zu Arbeitszeit und Arbeitsbedingungen sowie einigen sozialstatistischen Daten. Die niederländische Umfrage startete im Jahr 2001 auf Basis des zwischen Ge-

\footnotetext{
1 Siehe zu einer ersten Auswertung der im Jahr 2009 erhobenen Daten für Deutschland Bispinck et al 2010 a.
}

Reinhard Bispinck, Dr., Wissenschaftler im Wirtschafts- und Sozialwissenschaftlichen Institut (WSI) und Leiter des WSI-Tarifarchivs in der Hans-Böckler-Stiftung. e-mail: Reinhard-Bispinck@boeckler.de Heiner Dribbusch, Dr., Wissenschaftler im WSI in der Hans-Böckler-Stiftung. Arbeitsschwerpunkte: Arbeitsbeziehungen und Gewerkschaftspolitik. e-mail: Heiner-Dribbusch@boeckler.de Fikret Öz, Wissenschaftler im Institut Arbeit und Technik (IAT).

e-mail: oez@iat.eu

Kea Tijdens, Dr., Professorin für Frauenerwerbstätigkeit (Women's Employment) an der Erasmus Universität Rotterdam, Forschungskoordinatorin am Amsterdam Institute for Advanced Labour Studies (AIAS) und assoziierte Professorin an der Universität Amsterdam. e-mail:k.g.tijdens@uva.nl Maarten van Klaveren forscht am AIAS. e-mail:m.vanklaveren@uva.nI 
werkschaften und der Universität von Amsterdam vereinbarten Loonwijzer-Projektes (www.loonwijzer.nl), das organisatorisch unter dem Dach der WageIndicator-Stiftung in den Niederlanden angesiedelt wurde. Im Rahmen einer europäischen Erweiterung des Projektes beteiligt sich das WSI seit 2004 an den Aktivitäten der Stiftung und führt seitdem in Deutschland eine eigene laufende Online-Erhebung zu Einkommens- und Arbeitsbedingungen von Erwerbstätigen durch. Auf der Internetseite www.lohnspiegel.de steht ein Online-Fragebogen zur Verfügung, den alle Interessierten ausfüllen können.

Seit August 2009 wurde im Rahmen dieser Online-Umfragen in beiden Ländern eine Reihe gleich lautender Fragen zu den Folgen der aktuellen Wirtschaftskrise für die Beschäftigten und ihren Arbeitsund Einkommensbedingungen seit Anfang 2009 gestellt. Sie beziehen sich auf die wirtschaftliche Situation des Betriebes, die Entwicklung der Beschäftigung, die ergriffenen personalpolitischen Maßnahmen sowie die Entwicklung der Einkommen und der Arbeitsbedingungen. Die vorliegende Auswertung basiert auf rund 19.000 Datensätzen von abhängig Beschäftigten in Deutschland und rund 12.000 in den Niederlanden, die im Zeitraum von August 2009 bis Juni 2010 erhoben wurden (Tabelle 1; Tijdens/van Klaveren 2010). Aufgrund der Anlage als selbst gesteuerte OnlineErhebung ist keine Repräsentativität der Ergebnisse gegeben. Die bisherigen Erfahrungen mit dem Lohnspiegel-Datensatz zeigen aber, dass den Ergebnissen dennoch eine hohe Aussagekraft zukommt (Bispinck et al. 2010b; Steinmetz et al. 2009).

\section{Reichweite der Krise}

Zunächst interessiert die Reichweite der Wirtschaftskrise. Zu diesem Zweck wurde gefragt, wie sich aus Sicht der Beschäftigten die wirtschaftliche Situation des Betriebs zum Befragungszeitpunkt im Vergleich zu Anfang des Jahres 2009 entwickelt hat (Abbildung 2). In den Niederlanden ist der Anteil der krisenbetroffenen Betriebe größer als in Deutschland: $41 \%$ der niederländischen Befragten geben an, dass es ihrem Betrieb zum Befragungszeitpunkt schlechter ging als Anfang 2009, während

\section{Abb. 1: Wirtschaftliche Kennziffern für Deutschland und die Niederlande seit der Krise}

Bruttoinlandsprodukt, preisbereinigt saison- und arbeitstagebereinigt

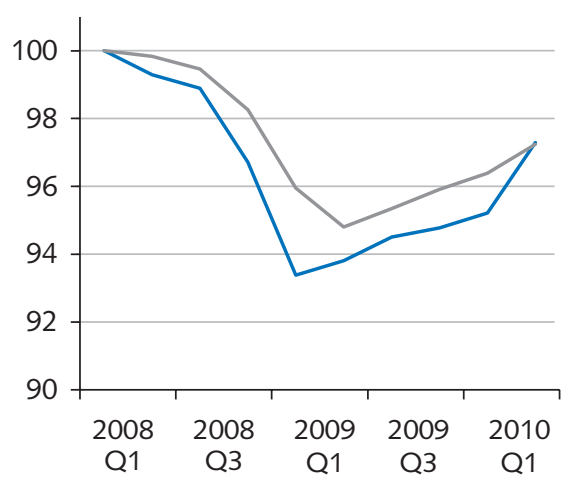

Arbeitsproduktivität je Person, saisonbereinigte Werte

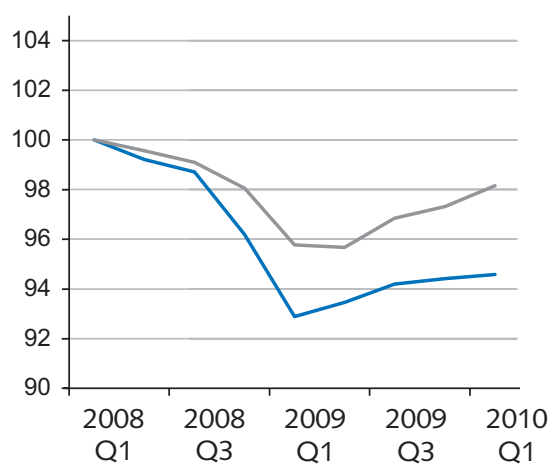

Arbeitslosenquote, harmonisiert, in $\%$

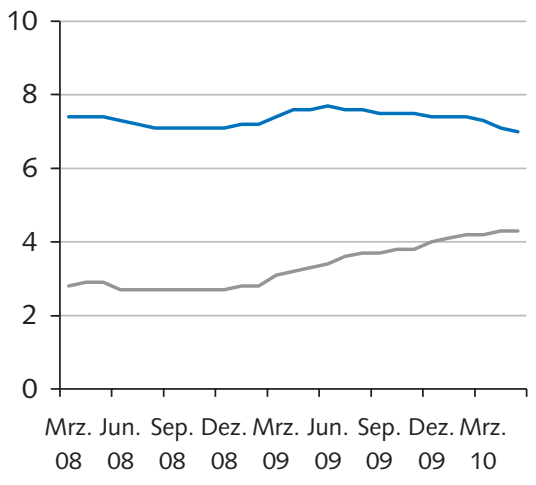

Lohnstückkosten, saisonbereinigte Werte

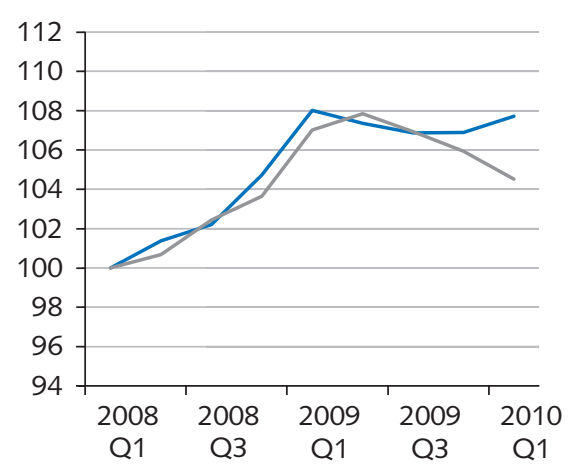

Deutschland

Quelle: Reuters Ecowin (Eurostat); Herzog-Stein et al. (2010)

WSI MITTELUNGEN

Tabelle 1: Datensatz der Online-Befragung Deutschland - Niederlande im Vergleich

\begin{tabular}{lcccc}
\hline & \multicolumn{2}{c}{ Deutschland } & \multicolumn{2}{c}{ Niederlande } \\
& absolut & $\%$ & absolut & $\%$ \\
\hline Gesamt & 18.847 & 100 & 12.289 & 100 \\
Männer & 12.347 & 65,5 & 6.082 & 57,5 \\
Frauen & 6.495 & 34,5 & 4.496 & 42,5 \\
Betriebsgröße & & & \\
unter 100 Beschäftigte & 9.678 & 51,4 & 7.493 & 61,0 \\
100-500 & 4.229 & 22,4 & 2.468 & 20,1 \\
über 500 & 4.939 & 26,2 & 2.328 & 18,9 \\
mit Tarifbindung & 8.514 & 51,1 & 7.718 & 71,2 \\
\hline $\begin{array}{l}\text { Quelle: www.lohnspiegel.de und www.loonwijzer.nl - } \\
\text { Befragungszeitraum: August 2009 - Juni 2010. }\end{array}$ & & W/SI MITEILUNGEN
\end{tabular}

das bei den deutschen Beschäftigten nur ein gutes Drittel (34\%) aussagt. In beiden Ländern äußern mehr als $40 \%$, dass die wirtschaftliche Lage gleich geblieben ist (D: $44 \%$, NL: $42 \%)$. Dementsprechend ist der Anteil der Betriebe, denen es nach Einschätzung der Beschäftigten zum Befragungszeitpunkt besser geht, in Deutsch- land mit $21 \%$ erkennbar höher als in den Niederlanden mit $17 \%$.

Die Betriebe, die sich nach Angabe der Beschäftigten in einer schlechteren wirtschaftlichen Situation als Anfang 2009 befanden, bezeichnen wir im Folgenden als „Krisenbetriebe“. ${ }^{2}$ Der Anteil dieser Krisenbetriebe ist im Befragungszeitraum 


\section{Abb. 2: Wirtschaftliche Lage der Betriebe, Deutschland und Niederlande*}

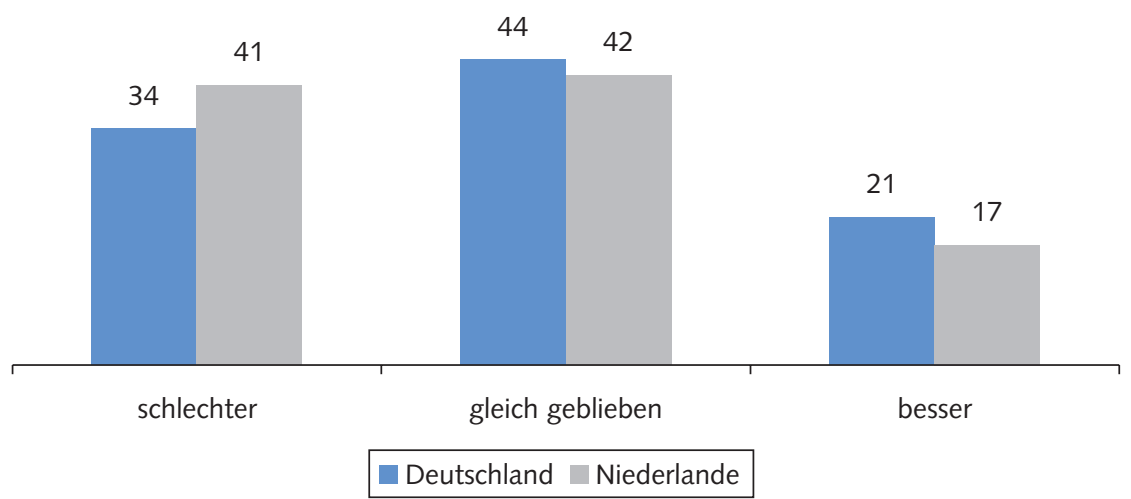

*Frage: Die wirtschaftliche Lage des Betriebs ist im Vergleich zum Jahresbeginn 2009 schlechter, gleich geblieben oder besser? Quelle: www.lohnspiegel.de und www. loonwijzer.nl, Befragungszeitraum: August 2009-Juni 2010.

WSI MITTELUNGEN

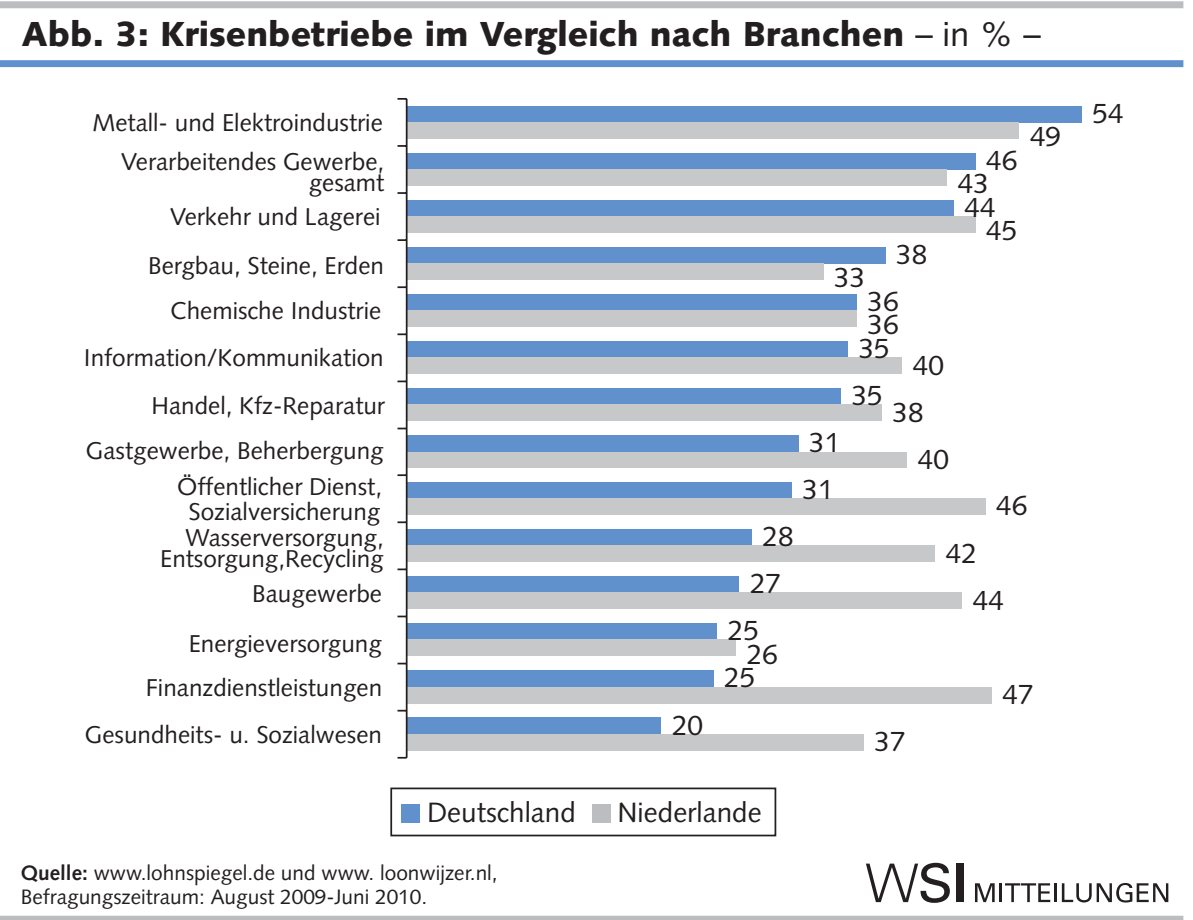

Tabelle 2: Beschäftigungsentwicklung in den Betrieben seit Anfang 2009 - Angaben der Befragten in \%, alle Betriebe -

\begin{tabular}{lcc} 
& Deutschland & Niederlande \\
\hline zurückgegangen & 39 & 40 \\
gleich geblieben & 40 & 40 \\
gestiegen & 21 & 20
\end{tabular}

Quelle: www.lohnspiegel.de und www.loonwijzer.nl Befragungszeitraum: August 2009 - Juni 2010.

WSI MiTTEILUNGEN

zurückgegangen: in Deutschland deutlich von $38 \%$ im dritten Quartal 2009 auf $29 \%$ im zweiten Quartal 2010, in den Niederlanden lediglich von $41 \%$ auf $40 \%$.

Mit Blick auf die Branchendifferenzierung zeigen sich teils Gemeinsamkei- ten, teils deutliche Unterschiede zwischen Deutschland und den Niederlanden (Abbildung 3). Die Metall- und Elektroindustrie wurde in beiden Ländern von der Krise am stärksten betroffen: $54 \%$ der deutschen und $49 \%$ der niederländischen Befragten aus diesem Wirtschaftsbereich geben an, dass es ihrem Betrieb schlechter geht als zu Beginn des Jahres 2009. Ähnlich sieht es im Bereich Verkehr und Speditionswesen aus mit $44 \%$ (D) und $45 \%$ (NL). In der chemischen Industrie fällt die Krisenbetroffenheit in beiden Ländern mit $36 \%$ gleich hoch aus. In der Energieversorgung konstatieren die Beschäftigten beider Länder mit 25 und $26 \%$ eine relativ niedrige Krisenbetroffenheit.

Weniger als ein Drittel Krisenbetriebe gibt es in Deutschland in sieben von zwölf Branchen, während in den Niederlanden lediglich zwei von zwölf Branchen eine solch niedrige Krisenbetroffenheit erkennen lassen. In der Mehrzahl der Branchen fällt die Einschätzung der Niederländer entsprechend deutlich schlechter aus als in Deutschland. Dies gilt vor allem für den Bereich Gesundheit und Sozialwesen (D: $20 \%$, NL: $37 \%$ ), für den Bereich Öffentlicher Dienst/Sozialversicherung (D: $31 \%$, NL: $46 \%$ ) und noch stärker für die Finanzdienstleistungen (D: $25 \%$, NL: 47 \%). Letzteres spiegelt die Tatsache wider, dass in den Niederlanden zwei der drei großen Finanzkonglomerate infolge der Krise in ernsthafte Schwierigkeiten gerieten. Alles in allem wurde die Krise somit in den Niederlanden von den Beschäftigten breiter wahrgenommen als in Deutschland, wo relativ weniger Unternehmen betroffen waren.

Die unterschiedliche wirtschaftliche Entwicklung der Betriebe beider Länder müsste sich in der Entwicklung der Beschäftigung niederschlagen. Erstaunlicherweise liegen zu Letzterer die Angaben der deutschen und niederländischen Befragten ganz dicht beieinander; signifikante Unterschiede sind nicht auszumachen (Tabelle 2). In etwa $40 \%$ der Betriebe ist im Vergleich zum Jahresbeginn 2009 die Beschäftigtenentwicklung zurückgegangen. In gleichem Umfang wird über eine konstante Beschäftigung berichtet. Und rund $20 \%$ geben an, die Beschäftigung sei gestiegen.

Wenn die jeweilige wirtschaftliche Lage der Betriebe berücksichtigt wird, bleiben zwar die Proportionen ähnlich, doch treten erkennbare Unterschiede auf. Die Krisenbetriebe in den Niederlanden weisen zu einem größeren Anteil (67\%)

2 Zu berücksichtigen ist, dass auch Betriebe, be denen gegenüber Jahresbeginn 2009 die ökonomische Situation unverändert blieb, krisenbetroffen sein können, wenn die ökonomische Verschlechterung bereits vor 2009 eingetreten ist. 
einen Beschäftigtenrückgang auf als in Deutschland (63\%). Anders dagegen in den Betrieben mit besserer wirtschaftlicher Lage: In den Niederlanden konstatieren die Beschäftigten zu einem deutlich geringeren Anteil (22 \%) einen Beschäftigungsrückgang als in Deutschland (30\%).

Die Einschätzungen von beschäftigten Männern und Frauen differieren in beiden Ländern bezüglich der wirtschaftlichen Lage der Betriebe besonders dann, wenn es um die Bewertung einer positiven Betriebsentwicklung geht. Hier sind offenbar Frauen in Deutschland spürbar pessimistischer und in den Niederlanden etwas skeptischer als Männer.

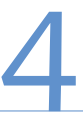

\section{Betriebliche Personalmaß- nahmen}

Die Beschäftigten beobachteten auch die konkreten Auswirkungen der Krise. Zunächst interessiert hier die Einschätzung von Maßnahmen zum Personalabbau. Insgesamt hat die Mehrheit aller Betriebe in beiden Ländern nach Angaben der Befragten in der Krise zu Maßnahmen des Personalabbaus gegriffen. Doch immerhin knapp ein Drittel (31 \%) der Betriebe in Deutschland und knapp $40 \%$ in den Niederlanden verzeichnen keinerlei Personalabbaumaßnahmen.

Das Bild wandelt sich, wenn man nur die Krisenbetriebe betrachtet (Abbildung 4). Mit $15 \%$ (D) bzw. $17 \%$ (NL) liegt der Anteil dieser Betriebe ohne Personalabbaumaßnahmen erheblich niedriger als im Durchschnitt aller Betriebe. Im Katalog der personalpolitischen Instrumente wurden „weiche“ Maßnahmen, die die Stammbelegschaften schonen, bevorzugt. In Deutschland liegt hier die Nicht-Wiederbesetzung von frei werdenden Stellen eindeutig an der Spitze (54\% der Betriebe), in den Niederlanden ist es das Auslaufen von befristeten Beschäftigungsverhältnissen (51 \%). Die Nutzungshäufigkeit fällt bei einigen Instrumenten in beiden Ländern relativ gleich aus. So z.B. bei der Kündigung von Leiharbeitern, bei der Kündigung von Stammpersonal und bei der Nicht-Übernahme von Auszubildenden. Deutliche Unterschiede gibt es bei der Kurzarbeit. Während dieses Instrument in Deutschland nach Angaben der Befragten in mehr als einem Drittel der Krisenbetriebe genutzt

\section{Abb. 4: Personalmaßnahmen in Krisenbetrieben - in \% -}

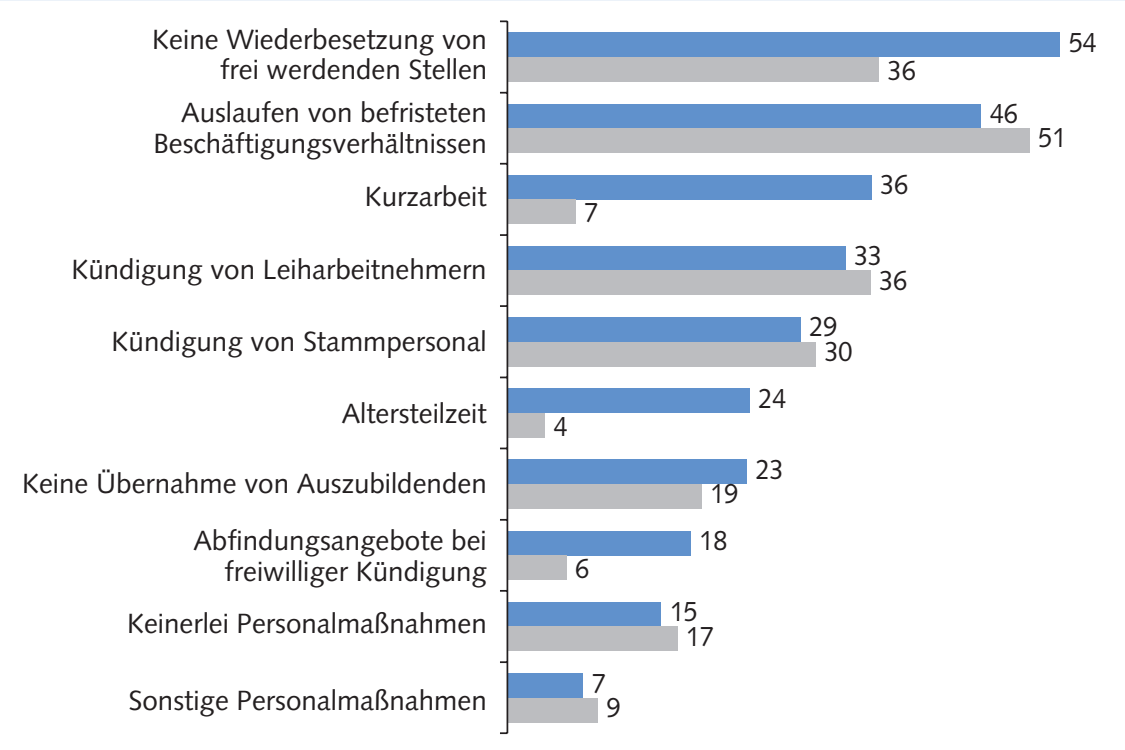

Deutschland Niederlande

Quelle: www.lohnspiegel.de und www. loonwijzer.nl,

Befragungszeitraum: August 2009 - Juni 2010.

WSI MITTEILUNGEN

wurde, fand in den Niederlanden ein vergleichbares Instrument, die sogenannte Teilzeit-Arbeitslosigkeit (deeltijd ww), nur in $7 \%$ der Betriebe Anwendung.

Zum Hintergrund: Das Instrument der Teilzeit-Arbeitslosigkeit wurde von der niederländischen Regierung während der Krise befristet eingeführt. Die Regelung kommt dann zum Tragen, wenn Beschäftigte mit mindestens $50 \%$ ihrer Arbeitszeit ,arbeitslos“ würden. Sie ist wesentlich restriktiver angelegt als ihr bundesdeutsches Pendant. Firmen mussten bis zu einer festgesetzten Frist, die in der ersten Jahreshälfte 2009 mehrfach verlängert wurde, Anträge einreichen, in denen eine ihre Existenz bedrohende wirtschaftliche Situation nachgewiesen werden musste. Etwa 600-800 Unternehmen/Firmen aus der Metall- und Chemieindustrie sowie der Bauwirtschaft, die teilweise mehrere Anträge stellten, konnten diese Regelung bislang in Anspruch nehmen. Auch wenn einige Firmen sicherlich mehr als einen Betrieb umfassten und wohl mehrere Anträge stellten, belegt dies eine im Vergleich zur deutschen Kurzarbeit deutlich geringere Nutzung dieses spezifisch niederländischen Arbeitsmarktinstruments. Die Kurzarbeit wurde 2009 im Jahresdurchschnitt immerhin von 55.800 deutschen Betrieben in Anspruch genommen.

Das niederländische „Kurzarbeitergeld“ gleicht $85 \%$ des auf die ausgefallene Ar- beitszeit entfallenden Entgelts (ohne eventuelle Zuschläge) aus. 2009 wurden jedoch viele Vereinbarungen zwischen Gewerkschaften und Unternehmen geschlossen, die eine Aufstockung des „Kurzarbeitergelds“ auf $100 \%$ des Einkommens vorsahen.

Sehr deutliche Unterschiede lassen sich auch in der Nutzung der Altersteilzeit feststellen (D: $24 \%$, NL: $4 \%$ ). Auch Abfindungsangebote bei freiwilliger Kündigung sind offenkundig in deutschen Betrieben (18\%) weiter verbreitet als in niederländischen (6 \%). Diese Ergebnisse decken sich in der Tendenz mit amtlichen statistischen Angaben der Bundesagentur für Arbeit bzw. des Ministerie van Sociale Zaken en Werkgelegenheid, nach denen beispielsweise in Deutschland im Jahr 2009 ca. $4 \%$ der Beschäftigten in Kurzarbeit waren gegenüber etwa $1 \%$ der niederländischen Beschäftigten, die sich in vergleichbaren Arrangements befanden.

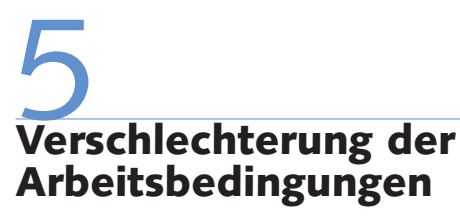

Häufig beklagen die Beschäftigten in beiden Ländern eine Verschlechterung der allgemeinen Arbeitsbedingungen. Besonders auffällig ist, dass bezogen auf alle Betriebe immerhin $68 \%$ der bundesdeutschen 
Tabelle 3: Arbeitsbedingungen in der Krise seit Anfang 2009, nach wirtschaftlicher Lage der Betriebe - Angaben der Beschäftigten in \%, alle Betriebe

\begin{tabular}{|c|c|c|c|c|c|c|}
\hline Wirtschaftliche Lage ist... & \multicolumn{2}{|c|}{..schlechter } & \multicolumn{2}{|c|}{..besser } & \multicolumn{2}{|c|}{..gleich geblieben } \\
\hline Auswirkung & $\mathrm{D}$ & $\mathrm{NL}$ & D & NL & D & $\mathrm{NL}$ \\
\hline Betriebsklima verschlechtert sich & 76 & 62 & 49 & 27 & 47 & 27 \\
\hline Aufstiegschancen verschlechtern sich & 66 & 73 & 39 & 21 & 40 & 28 \\
\hline Zeitdruck nimmt zu & 68 & 57 & 57 & 41 & 51 & 37 \\
\hline Leistungsdruck nimmt zu & 77 & 67 & 65 & 52 & 61 & 44 \\
\hline
\end{tabular}

und $54 \%$ der niederländischen Umfrageteilnehmer über eine Zunahme des Leistungsdrucks berichten. Einen gestiegenen Zeitdruck stellen $58 \%$ der Beschäftigten in Deutschland und $46 \%$ der Befragten aus den Niederlanden fest. Beides geht Hand in Hand mit sich verschlechternden Aufstiegschancen und einer Verschlechterung des Betriebsklimas.

Dass in Betrieben, deren wirtschaftliche Lage sich verschlechtert hat, das Betriebsklima besonders häufig leidet, kann nicht wirklich überraschen (Tabelle 3). In Deutschland stellen dies gut drei Viertel aller Befragten aus diesen Betrieben fest (76\%). In den Niederlanden liegt der entsprechende Wert deutlich niedriger (62 \%). Die Verschlechterung des Betriebsklimas ist im Vergleich von Krisenbetrieben zu denen mit besserer oder gegenüber 2008 unveränderter Wirtschaftslage (jeweils „nur“ $27 \%$ ) besonders markant. Mit $77 \%$ ist der Anteil derer in den Krisenbetrieben, die einen erhöhten Leistungsdruck feststellen, in Deutschland besonders ausgeprägt; aber auch in den Niederlanden wird dies in immerhin zwei

Drittel aller Krisenbetriebe konstatiert. Auffallend ist, dass in beiden Ländern der Zeit- und Leistungsdruck in den Betrieben mit verbesserter wirtschaftlicher Lage häufiger gestiegen ist als in jenen, deren wirtschaftliche Situation als unverändert eingestuft wird. Die in Deutschland beschäftigten Frauen geben in allen vier abgefragten Dimensionen etwas häufiger Verschlechterungen zu Protokoll als die Männer. In den Niederlanden ist das diesbezügliche Bild insgesamt ausgeglichener.

\section{Auswirkungen auf Einkommen}

Die Krise wirkte sich - wie zu erwarten war - negativ auf die Einkommen der Beschäftigten aus. Betroffen sind vor allem Monatseinkommen und Sonderzahlungen wie Weihnachtsgeld, Urlaubsgeld sowie weitere Zulagen. In Deutschland geben $34 \%$ der Befragten in unserem Datensatz an, dass sie Einkommensverluste zu

\section{Abb. 5: Auswirkungen der Wirtschaftskrise auf die Einkommen}

- Angaben der Beschäftigten in \%, alle Betriebe -

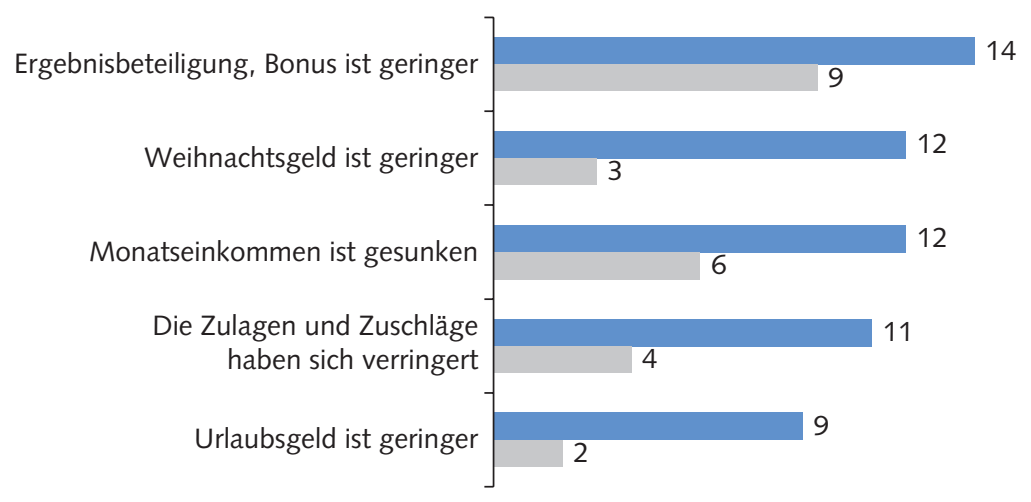

Deutschland Niederlande

Quelle: www.lohnspiegel.de und www. loonwijzer.nl,

Befragungszeitraum: August 2009-Juni 2010 verzeichnen hatten, in den Niederlanden „nur“ rund $17 \%$. Das Ausmaß, in dem negative Auswirkungen seitens der Beschäftigten berichtet werden, differiert allerdings zum Teil erheblich: So antworteten auf die Frage, welche Auswirkungen die Wirtschaftskrise für sie persönlich habe - bezogen auf alle Betriebe - $12 \%$ der Beschäftigten in Deutschland, aber nur $6 \%$ der Niederländer, dass ihr Monatseinkommen gesunken sei (Abbildung 5). Die Ursachen für diese Differenzen lassen sich aus den Umfrageergebnissen nicht erschließen. Zu vermuten ist, dass in der Bundesrepublik im Vergleich zu den Niederlanden die insgesamt relativ weite Verbreitung von Kurzarbeit, aber auch Einschnitte im Zusammenhang mit betrieblichen Krisenvereinbarungen das Ergebnis prägen.

Einschnitte bei Zulagen und Zuschlägen wurden mit $11 \%$ beinahe dreimal häufiger, Kürzungen bei Weihnachtsgeld und Urlaubsgeld mit $12 \%$ bzw. $9 \%$ sogar ca. viermal häufiger von deutschen Beschäftigten als von niederländischen festgestellt (3\% bzw. $2 \%$ ). Am häufigsten gab es in beiden Ländern Einschnitte bei Ergebnisbeteiligungen oder Boni. Auch hier lag aber der entsprechende Anteil in den Niederlanden mit $9 \%$ deutlich unter dem entsprechenden Wert in Deutschland (14\%).

Diese Unterschiede bleiben auch bestehen, wenn wir die Ergebnisse der Umfrage unter dem Blickwinkel der Krisenbetroffenheit der jeweiligen Betriebe ansehen (Tabelle 4). In den Betrieben, deren wirtschaftliche Lage sich gegenüber Jahresbeginn 2009 verschlechtert hat, sind die Folgen deutlicher zu spüren: Gut jeder fünfte Beschäftigte gibt in Deutschland an, dass das Monatseinkommen gesunken sei. Dieser Wert liegt erheblich über den Ergebnissen aus Betrieben, deren wirtschaftliche Lage als besser oder gleich geblieben eingeschätzt wird. In den Niederlanden sind die entsprechenden Werte überall niedriger, auch hier gibt es aber in den Krisenbetrieben mit $8 \%$ erheblich häufiger Rückmeldungen über Einschnitte beim Monatseinkommen als in den anderen Betrieben. In seiner Tendenz ähnlich ist das Bild zu den berichteten Einschnitten bei den Jahressonderzahlungen (Urlaubs- und Weihnachtsgeld).

Abweichend und auf den ersten Blick verblüffend sind dagegen zum Teil die Ergebnisse bezüglich Zulagen, Zuschlägen 
und Ergebnisbeteiligungen. Auffallend ist insbesondere, dass in Deutschland der Anteil der Beschäftigten, die über Einschnitte bei Zulagen und Zuschlägen berichten, in den Krisenbetrieben mit $17 \%$ annähernd gleich hoch ausfällt wie in den Betrieben, deren wirtschaftliche Lage sich verbessert hat (16\%). In den Niederlanden liegt der entsprechende Anteil in den Krisenbetrieben sogar leicht unter dem der Betriebe mit verbesserter wirtschaftlicher Lage. Die wenigsten Einschnitte bei den Zulagen gab es in den Betrieben, deren Situation in Folge der Krise als unverändert eingeschätzt wurde. Über die Ursachen lässt sich auf Basis der Umfrage keine Aussage treffen; doch liegt die Vermutung nahe, dass nicht nur in Krisenbetrieben, sondern auch in einer Reihe von Betrieben, die ihre wirtschaftliche Lage verbessern konnten, Spar- und Kürzungsprogramme umgesetzt wurden. Auch bei Ergebnisbeteiligungen und Bonuszahlungen fallen in Deutschland die relativ hohen Werte bezüglich der Einschnitte in Betrieben mit verbesserter wirtschaftlicher Lage auf, die deutlich über denen in Betrieben mit unveränderter Wirtschaftslage liegen. Dagegen zeigt sich in den Niederlanden das vordergründig zu erwartende Bild. In den Krisenbetrieben wurden hier doppelt so häufig Einschnitte gemeldet wie in Betrieben mit besserer oder gleich gebliebener Lage.

Von negativen Auswirkungen auf die Einkommen sind Frauen in Deutschland etwas seltener betroffen als Männer (63\% bzw. $59 \%$ ). Insbesondere Rückgänge bei den Monatseinkommen sowie Kürzungen bei Zulagen und Zuschlägen kommen bei ihnen weniger häufig vor. Von Kürzungen bei den Jahressonderzahlungen und Ergebnisbeteiligungen sind Frauen in Deutschland ebenfalls seltener betroffen, besonders deutlich ist die unterschiedliche Betroffenheit bei den Bonuskürzungen. In den Niederlanden bietet sich im Vergleich hierzu ein anderes Bild. Dort geben Frauen deutlich häufiger als Männer an, dass ihr Monatseinkommen gesunken ist. Bei Zulagen und Jahressonderzahlungen in den Niederlanden gibt es dagegen nur geringe oder keine Unterschiede zwischen den Geschlechtern; lediglich bei den Ergebnisbeteiligungen und Boni berichten Frauen seltener von Kürzungen als ihre männlichen Kollegen.
Tabelle 4: Auswirkungen der Krise auf die Einkommen, nach Wirtschaftslage der Betriebe

- Angaben der Beschäftigten in \%, alle Betriebe -

\begin{tabular}{|c|c|c|c|c|c|c|}
\hline \multirow{2}{*}{$\begin{array}{c}\text { Wirtschaftliche Lage ist... } \\
\text { Auswirkung }\end{array}$} & \multicolumn{2}{|c|}{..schlechter } & \multicolumn{2}{|c|}{..besser } & \multicolumn{2}{|c|}{..gleich geblieben } \\
\hline & D & NL & D & NL & D & NL \\
\hline Monatseinkommen gesunken & 21 & 8 & 9 & 4 & 7 & 3 \\
\hline $\begin{array}{l}\text { Zulagen und Zuschläge haben sich } \\
\text { verringert }\end{array}$ & 17 & 6 & 16 & 7 & 7 & 2 \\
\hline $\begin{array}{l}\text { Ergebnisbeteiligung, Bonuszahlung ist } \\
\text { geringer }\end{array}$ & 21 & 14 & 16 & 7 & 9 & 6 \\
\hline Weihnachtsgeld ist geringer & 18 & 5 & 10 & 2 & 9 & 2 \\
\hline Urlaubsgeld ist geringer & 13 & 3 & 7 & 2 & 7 & 1 \\
\hline
\end{tabular}

\section{7 Beschäftigungsentwicklung
in geschlechtsspezifischer
Wahrnehmung}

Geht es um die Beschäftigungsentwicklung, so lassen sich in beiden Ländern kaum geschlechtsspezifische Unterschiede in der Häufigkeit feststellen, mit der ein Anstieg der Beschäftigung konstatiert wird (Tabelle 5). Etwas markanter sind die Unterschiede mit fünf Prozentpunkten lediglich bezüglich der Häufigkeit, mit der Männer und Frauen in Deutschland einen Beschäftigungsrückgang beziehungsweise eine unveränderte Personalsituation in ihren Betrieben feststellen.

Ist die generelle Einschätzung der Beschäftigungsentwicklung aus der Geschlechterperspektive noch annähernd gleich, so gehen die Auffassungen darüber, ob Frauen besonders vom Beschäftigungsabbau betroffen sind, zwischen Männern und Frauen derart weit auseinander, dass von fundamental unterschiedlichen Wahrnehmungen der geschlechtsspezifischen Dimension des Personalabbaus ausgegangen werden muss.

Dies gilt in besonders extremer Weise für die Niederlande, wo lediglich $8 \%$ der Männer, aber mit $46 \%$ fast die Hälfte der weiblichen Beschäftigten der Einschätzung sind, dass Frauen seit Beginn 2009 beson- ders vom Personalabbau betroffen waren. In Deutschland ist die Differenz der Werte (Männer: $15 \%$, Frauen: $41 \%$ ) graduell geringer, aber mit 26 Prozentpunkten ebenfalls ganz beträchtlich. Diese Unterschiede in der Wahrnehmung zeigen sich auch, wenn der Frauenanteil der jeweiligen Betriebe berücksichtigt wird. In ausgesprochen männerdominierten Betrieben - hier definiert als solche, in denen der Frauenanteil lediglich zwischen 10 und $20 \%$ der Beschäftigten liegt - sehen in den Niederlanden immerhin $16 \%$ der Frauen, aber nur 4 \% der Männer, Frauen als besonders vom Personalabbau betroffen. In Deutschland teilen $11 \%$ der Männer und $20 \%$ der Frauen aus Männerbetrieben diese Ansicht. In Betrieben mit einem Frauenanteil von $80 \%$ und mehr nehmen in den Niederlanden immerhin $60 \%$ der Männer eine besondere Betroffenheit von Frauen wahr, bei den Frauen sind es hier $91 \%$. In Deutschland ist in diesen Betrieben die Wahrnehmungsdifferenz mit $44 \%$ der Männer und $67 \%$ der Frauen ebenfalls deutlich ausgeprägt.

\section{Welche Beschäftigten sind von Einkommensverlusten besonders betroffen?}

Die deskriptiven Analysen zeigen, dass die Höhe und Intensität von krisenbedingten
Tabelle 5: Betriebliche Beschäftigungsentwicklung

- Angaben der Beschäftigten in \%, alle Betriebe -

\begin{tabular}{|c|c|c|c|c|}
\hline \multirow{2}{*}{$\begin{array}{l}\text { Seit Anfang } 2009 \text { ist die Zahl } \\
\text { der Beschäftigten im Betrieb... }\end{array}$} & \multicolumn{2}{|c|}{ Deutschland } & \multicolumn{2}{|c|}{ Niederlande } \\
\hline & Männer & Frauen & Männer & Frauen \\
\hline ..zurückgegangen & 40 & 35 & 41 & 39 \\
\hline ..gleich geblieben & 39 & 44 & 39 & 41 \\
\hline ..gestiegen & 21 & 21 & 21 & 20 \\
\hline
\end{tabular}


Tabelle 6: Wahrscheinlichkeit der Betroffenheit von krisenbedingten Einkommensverlusten (Logistische Regression)

\begin{tabular}{|c|c|c|c|c|c|c|c|}
\hline \multirow{2}{*}{ Betriebliche Faktoren } & & \multicolumn{3}{|c|}{ Deutschland } & \multicolumn{3}{|c|}{ Niederlande } \\
\hline & & $\begin{array}{l}\text { Regressions- } \\
\text { koeffizient B }\end{array}$ & Sign. & $\operatorname{Exp}(B)$ & $\begin{array}{l}\text { Regressions- } \\
\text { koeffizient B }\end{array}$ & Sign. & $\operatorname{Exp}(B)$ \\
\hline \multirow[t]{3}{*}{ Betriebsgröße } & unter 100 (Referenzgruppe) & & $* * *$ & & & & \\
\hline & $100-500$ & ,016 & & 1,016 &,- 037 & & ,963 \\
\hline & über 500 & ,195 & $* * *$ & 1,215 & ,142 & & 1,152 \\
\hline \multirow[t]{5}{*}{ Wirtschaftszweige } & Verarbeitendes Gewerbe (Referenz) & & $* * *$ & & & $* * *$ & \\
\hline & Bau, Energie-, Wasserversorgung &,- 619 & $* * *$ & ,539 &,- 506 & $* * *$ &, 603 \\
\hline & Handel, Verkehr, Gastgewerbe, luk &,- 405 & $* * *$ & ,667 &,- 274 & $* * *$ & ,761 \\
\hline & Wirtschaftliche Dienstleistungen &,- 381 & $* * *$ & ,683 &,- 169 & & ,844 \\
\hline & $\begin{array}{l}\text { Öffentliche Verwaltung, } \\
\text { Gesundheit, Bildung }\end{array}$ &,- 667 & $* * *$ &, 513 &,- 712 & $* * *$ & ,490 \\
\hline \multirow[t]{2}{*}{ Tarifbindung im Betrieb } & Ohne Tarifbindung (Referenz) & & & 1,000 & & & 1,000 \\
\hline & Mit Tarifbindung &,- 109 & * & ,897 &,- 358 & $* * *$ & ,699 \\
\hline \multirow{3}{*}{$\begin{array}{l}\text { Wirtschaftsentwicklung } \\
\text { gegenüber Anfang } 2009\end{array}$} & schlechter (Referenz) & & $* * *$ & 1,000 & & $* * *$ & 1,000 \\
\hline & gleich geblieben &,- 776 & $* * *$ & ,460 &,- 719 & $* * *$ & ,487 \\
\hline & besser &,- 600 & $* * *$ &, 549 &,- 667 & $* * *$ &, 513 \\
\hline Im Betrieb wurden & Trifft nicht zu (Referenz) & & & 1,000 &, 827 & $* * *$ & 1,000 \\
\hline $\begin{array}{l}\text { Personalmaßnahmen } \\
\text { durchgeführt }\end{array}$ & Trifft zu & ,990 & $* * *$ & 2,691 & & & 2,286 \\
\hline \multicolumn{8}{|l|}{ Persönliche Faktoren } \\
\hline \multirow[t]{2}{*}{ Geschlecht } & Frau (Referenz) & & & 1,000 & & & 1,000 \\
\hline & Mann &,- 364 & $* * *$ & 1,439 &,- 235 & $* *$ & 1,265 \\
\hline \multirow[t]{4}{*}{ Altersklassen } & Unter 30 (Referenz) & & $* * *$ & 1,000 & & & 1,000 \\
\hline & 30 bis unter 40 & ,122 & $*$ & 1,130 & ,004 & & 1,004 \\
\hline & 40 bis unter 50 & ,403 & $* * *$ & 1,497 &, 076 & & 1,079 \\
\hline & Über 50 &, 527 & $* * *$ & 1,693 &,- 113 & & ,894 \\
\hline \multirow[t]{3}{*}{ Abgeschlossene Schulbildung } & Höheres Bildungsniveau (Referenz) & & $* * *$ & 1,000 & & & 1,000 \\
\hline & Niedrigeres Bildungsniveau & ,203 & $* * *$ & 1,224 & ,113 & & 1,120 \\
\hline & Mittleres Bildungsniveau & ,008 & & 1,008 &, 060 & & 1,062 \\
\hline \multirow[t]{2}{*}{ Unbefristeter Arbeitsvertrag } & Trifft nicht zu (Referenz) & & & 1,000 & & & 1,000 \\
\hline & Trifft zu & 159 & * & 1,173 & 097 & & 1,102 \\
\hline \multirow[t]{2}{*}{ Arbeitszeit } & Teilzeit (Referenz) & & & & & & \\
\hline & Vollzeit & ,154 & $*$ & 1,167 &, 042 & & 1,043 \\
\hline Konstante & & $-1,104$ & $* * *$ & ,332 & $-1,334$ & $* * *$ & ,263 \\
\hline $\mathrm{N}$ & & 13.397 & & & 8.065 & & \\
\hline Nagelkerkes $\mathrm{R}^{2}$ & &, 17 & & & , 10 & & \\
\hline -2-log-likelihood & & $15.470,768$ & & & $6.895,607$ & & \\
\hline Chi² df(18) & & $1.789,980$ & & & 511,339 & & \\
\hline
\end{tabular}

Signifikanzniveau: * $p<0,05, * * p<0,01, * * * p<0,001$

Lesehilfe: Die Referenzgruppe für die Einflussvariable „Geschlecht“ bilden Frauen und der Exp(b) beträgt 1,439 für Deutschland.

Dies bedeutet, dass sich das Risiko von Einkommensverlusten in der Krise bei Männern gegenüber Frauen um 44 \% erhöht:

$(=(1,439-1) * 100)$. Bei einem Koeffizienten unter eins wie bei der Tarifbindung verringert sie sich dagegen um $10 \%(=(0,897-1) * 100)$.

Quelle: www.lohnspiegel.de und www.loonwijzer.nl; Berechnungen der Autoren.

Einkommensverlusten nach betrieblichen und persönlichen Faktoren variieren. Inwieweit solche Faktoren Einkommensverluste auch bei Kontrolle der Wechselwirkung untereinander beeinflussen, wurde mittels einer logistischen Regression untersucht. Bei dieser multivariaten Methode wird ein Wahrscheinlichkeitsmodell für das Eintreten und Nicht-Eintreten eines Ereignisses in Abhängigkeit von erklärenden Variablen modelliert (vgl. Fromm 2010). In die Analyse einbezogen wurden betriebsrelevante Faktoren wie Betriebsgröße, Wirtschaftszweige, Tarifbindung und Betroffenheit und Durchführung von Krisenmaßnahmen in den Betrieben. Persönliche Faktoren beziehen sich auf Geschlecht, Alter, Bildungsniveau, Arbeitszeit und Arbeitsvertrag. Die Ergebnisse der lo- gistischen Regression sind in der Tabelle 6 ausgewiesen. Sie zeigen, dass das Risiko in Bezug auf Einkommensverluste sowohl von betrieblichen als auch persönlichen Faktoren beeinflusst wird, die allerdings generell in den Niederlanden weniger ausgeprägt zum Tragen kommen als in Deutschland.

In Bezug auf die betriebsrelevanten Faktoren zeichnet sich im Ländervergleich ein differenziertes Bild ab. Im Vergleich zu kleineren Betrieben (unter 100 Beschäftigte) ist in größeren Betrieben die Wahrscheinlichkeit von Einkommensverlusten deutlich höher. Mit Ausnahme der Betriebe mittlerer Größe in den Niederlanden (100-500 Beschäftigte) gilt dies für beide Länder. Auch die Branchen haben einen großen Effekt: Im Vergleich zum verarbei- tenden Gewerbe ist die Wahrscheinlichkeit, von Einkommensverlusten betroffen zu sein, in allen anderen Wirtschaftszweigen niedriger. Die Unterschiede zwischen den Branchen sind in Deutschland stärker als in den Niederlanden. Dies steht im Einklang mit der Schlussfolgerung des deskriptiven Teils: In Deutschland sind anteilsmäßig weniger Wirtschaftszweige von der Krise betroffen als in den Niederlanden. Auffallend sind die Ergebnisse für den Bereich „Wirtschaftliche Dienstleistungen“. Im Vergleich zur Referenzkategorie „Verarbeitendes Gewerbe“ verringert sich das Wahrscheinlichkeitsverhältnis „Einkommensverluste/keine Verluste“ in Deutschland um $32 \%$ und in den Niederlanden um $17 \%$. Eine Tarifbindung des Betriebs verringert in beiden Ländern die 
Wahrscheinlichkeit, Einkommensverluste zu erleiden.

Die Beschäftigten in den Betrieben, die wegen der Krise bereits Personalmaßnahmen durchgeführt haben, weisen ein höheres Risiko von Einkommensverlusten auf. Dies gilt in beiden Ländern, allerdings in Deutschland stärker ausgeprägt als in den Niederlanden. In Deutschland steigt das Risiko um den Faktor 2,7 und in den Niederlanden um den Faktor 2,3. Wie erwartet ist das Risiko von Einkommensverlusten in den Krisenbetrieben höher als in den Betrieben, in denen die wirtschaftliche Entwicklung seit Anfang 2009 gleich geblieben oder besser verlaufen ist. In den Betrieben mit besserer Wirtschaftsentwicklung verringert sich das Risiko in Deutschland um $45 \%$ und in den Niederlanden um $48 \%$.

Was die persönlichen Merkmale betrifft, so steigt vor allem in Deutschland das Risiko von Einkommensverlusten mit dem Alter. In den Niederlanden fällt dieser Effekt nicht ins Gewicht. Männer haben im Vergleich zu Frauen ein höheres Risiko, Einkommensverluste hinnehmen zu müssen. Auch dieser Effekt ist in Deutschland stärker ausgeprägt als in den Niederlanden.

Schließlich hat auch der Bildungsabschluss Einfluss auf das Einkommensrisiko. Beschäftigte mit niedrigeren Bildungsabschlüssen weisen eine größere Wahrscheinlichkeit von Einkommensverlusten auf als Beschäftigte mit mittlerem und höherem Bildungsniveau. Dieser
Effekt ist wiederum in den Niederlanden weniger ausgeprägt. Auch Vollzeitbeschäftigte und Beschäftigte mit einem unbefristeten Arbeitsvertrag tragen hier ein höheres Risiko.

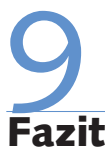

Die Wirtschaftskrise und ihre Folgen für Beschäftigung und Einkommen aus Sicht der Beschäftigten sind in der bisherigen Forschung zu kurz gekommen. Auf der Basis einer breit angelegten, über nahezu ein Jahr durchgeführten Onlineerhebung, an der sich rund 31.000 Beschäftigte beteiligt haben, wurde hier für Deutschland und die Niederlande analysiert, wie die Beschäftigten in den beiden Ländern die Auswirkungen auf die Betriebe und ihre persönlichen Arbeits- und Einkommensbedingungen beurteilen.

Zusammenfassend lässt sich festhalten: In den Niederlanden waren deutlich mehr Betriebe von der Krise betroffen als in Deutschland. Dies liegt vor allem daran, dass in Deutschland offenbar weniger Branchen ausgeprägt von der Krise betroffen waren als in den Niederlanden. Dort wurden anders als in Deutschland neben der Industrie u. a. auch das Baugewerbe, die Finanzdienstleistungen und die Bereiche Gesundheit und Soziales sowie Öffent- licher Dienst und Sozialversicherung als stark krisenbetroffen genannt.

Umgekehrt ergriffen die Krisenbetriebe in den Niederlanden nach Beobachtung der Beschäftigten seltener zu Maßnahmen der Personalanpassung. Bei dem Bündel der genutzten Instrumente zeigen sich ebenfalls Unterschiede: Kurzarbeit und Altersteilzeit wurden in den Niederlanden anders als in Deutschland kaum genutzt; und auch freiwillige Kündigungen verknüpft mit Abfindungsangeboten blieben dort die absolute Ausnahme.

Auch Einkommenseinbußen wurden in Deutschland von den Beschäftigten im Zusammenhang mit der Krise deutlich häufiger konstatiert als in den Niederlanden. Kürzungen des Monatseinkommens wurden von den Beschäftigten in Deutschland doppelt so oft genannt, Einschnitte bei Zulagen und Zuschlägen sowie bei Sonderzahlungen drei- bis viermal so oft. Bei Krisenbetrieben fielen die Abstände sogar noch größer aus.

Die multivariate Analyse hat gezeigt, dass neben den betriebsrelevanten Faktoren (vor allem die Branchenzugehörigkeit) auch personenbezogene Merkmale (Geschlecht, Alter, Qualifikation) Auswirkungen auf die Verteilung des krisenbedingten Einkommensrisikos haben, die allerdings in Deutschland stärker ausfallen als in den Niederlanden.

\section{LITERATUR}

Bispinck, R./Dribbusch, H./Öz, F. (2010a): Auswirkungen der Wirtschaftskrise auf die Beschäftigten, Ergebnisse der Online-Erhebung des Projekts LohnSpiegel, WSI-Report 2, Düsseldorf

Bispinck, R./Dribbusch, H./Öz, F. (2010b): Das Projekt LohnSpiegel: Tatsächlich gezahlte Löhne und Gehälter, in: WSI-Mitteilungen 63 (1), S. $42-49$

Bogedan, C./Brehmer, W./Herzog-Stein, A. (2009): Betriebliche Beschäftigungssicherung in der Krise - Eine Kurzauswertung der WSIBetriebsrätebefragung 2009, WSI-Report 1, Düsseldorf

Fromm, S. (2010): Datenanalyse mit SPSS für Fortgeschrittene 2: Multivariate Verfahren für Querschnittsdaten, Wiesbaden
Herzog-Stein, A./Lindner, F./Sturn, S./van Treeck, T. (2010): Vom Krisenherd zum Wunderwerk? Der deutsche Arbeitsmarkt im Wandel, IMK Report 56, Düsseldorf

Steinmetz, S./Tijdens, K.G./De Pedraza García, P. (2009): Comparing different weighting procedures for volunteer web surveys: Lessons to be learned from German and Dutch Wagelndicator data, AIAS Working Paper 76, Amsterdam

Tijdens, K.G./Van Klaveren, M. (2010): Crisis vragen in de Loonwijzer web-survey 2009, AIAS, Rapport voor FNV Bondgenoten, Amsterdam 